\title{
A new equation of state with abundances of all nuclei in core collapse simulations of massive stars
}

\author{
Shun Furusawa*1, Kohsuke Sumiyoshi ${ }^{2}$, Shoichi Yamada ${ }^{1,3}$, Hideyuki Suzuki ${ }^{4}$ \\ ${ }^{1}$ Department of Science and Engineering, Waseda University, 3-4-1 Okubo, Shinjuku, Tokyo \\ 169-8555, Japan \\ ${ }^{2}$ Numazu College of Technology, Ooka 3600, Numazu, Shizuoka 410-8501, Japan \\ ${ }^{3}$ Advanced Research Institute for Science and Engineering, Waseda University, 3-4-1 Okubo, \\ Shinjuku, Tokyo 169-8555, Japan \\ ${ }^{4}$ Faculty of Science and Technology, Tokyo University of Science, Yamazaki 2641, Noda, Chiba \\ 278-8510, Japan \\ E-mail: furusawa@heap.phys.waseda.ac.jp
}

\begin{abstract}
We construct the equations of state for baryons at sub-nuclear densities for the use in core-collapse simulations of massive stars. The abundance of various nuclei is obtained together with the thermodynamic quantities. The formulation is the NSE description and the liquid drop approximation of nuclei. The model free energy to minimize is calculated by relativistic mean field theory for nucleons and the mass formula for nuclei with the atomic number up to $\sim 1000$. We have also taken into account the pasta phase for heavy nuclei and the contribution of Pauli energies between free nucleons to binding energies to light nuclei. The experimental and theoretical mass data are employed to evaluate the shell effects of nuclei. We find that the abundance of heavy nuclei is different depending on shell effects of nuclei, which may have an important effect to the rates of electron captures and coherent neutrino scatterings on nuclei in supernova cores. The Pauli and self energy shifts also affect the abundance of light nuclei by comparing with ordinary nuclear statistical equilibrium results, which may affect the heating and cooling rates of supernova cores and shocked envelopes.
\end{abstract}

XII International Symposium on Nuclei in the Cosmos,

August 5-12, 2012

Cairns, Australia

${ }^{*}$ Speaker. 


\section{Introduction}

Core collapse supernovae occur at the end of the evolution of massive stars. The mechanism of this events is not clearly understood because of the complication. One of the underlying problem is equations of state (EOS's) of hot and dense matter both at sub- and supra-nuclear densities. EOS provides information on compositions of nuclear matter in addition to thermodynamical quantities such as pressure, entropy and sound velocities. The compositions play important roles at both pre- and post- bounce phases. In collapsing cores, they have an influence on the rate of electron captures and neutrino coherent scatterings on nuclei, both of which determine the evolution of lepton fraction, one of the most critical ingredients for the core dynamics. After bounce they affect the rates of heating and cooling process through the neutrino emission and absorption of nucleons and nuclei. [1, 2]

At present, there are only two EOSs in wide use for the simulations of core-collapse supernovae. Lattimer-Swesty's EOS is based on Skyrme-type nuclear interactions and the so called compressible liquid drop model for nuclei surrounded by dripped nucleons]3. The EOS by Shen et al. employs a relativistic mean fieeld theory (RMF) to describe nuclear matter and the ThomasFermi approximation for finite nuclei with dripped nucleons [4,56]. It should be emphasized here that both EOSs adopt the so-called single nucleus approximation (SNA), in which only a single representative nucleus is included. In other words, the distribution of nuclei is ignored. In this decade, some EOS's including multi-nuclei are researched by some research groups. All models assumed NSE but the description of nuclei at high temperatures and densities are different. Botvina's EOS [7] 8] is a generalization of the statistical model, which is one of the most successful models used for the theoretical description of multifragmentation [9], for astrophysical conditions. The nuclei of this model are based on the liquid drop model with the mass number up to 1000. However they ignored the shell effects of nuclei, which is important for reproducing the abundance of nuclei at low temperatures, On the other hands, EOS's of Hempel et al. [10] adopted masses of nuclei from two mass tables, which are experimental and theoretical mass data for isolated nuclei. They ignored the heavier nuclei with proton number $Z \gtrsim 100$ owing to the limitation of the mass tables and the high density and temperature effects on nuclear binding energies. G. Shen et al. [11] employs different theories, the Virial expansion at low densities and SNA with the Hartree approximation at high densities. The multi nuclei description are assumed in only low density regime and some quantities such as free proton fraction are discrete at the transition between the two descriptions (multi nuclei and the SNA).

We modeled a EOS [12] based on the NSE description using the mass formula for nuclei up to the atomic number of 1000 under the influence of surrounding nucleons and electrons. The mass formula is based on experimental data of nuclear binding energies that allow us to take into account nuclear shell effects. An extended liquid drop model is used to describe the medium effects, and in particular, formation of the pasta phases. Because of this combination, the free energy of a multicomponent system can reproduce the ordinary NSE results at low densities and make a continuous transition to the EOS for supra-nuclear densities. The details of models and comparisons with $\mathrm{H}$. Shen's EOS and Hempel's EOS are given in [12]. The purpose of this study is to construct more realistic EOS for the core-collapse supernova simulations. In this proceedings, we report on our new EOS and discuss the difference from the previous one. 


\section{Our new models}

To obtain the multi-component EOS's we construct a model of free energy and minimize it with respect to the parameters included in the free energy. The matter in the supernova core at sub-nuclear densities consists of nucleons, nuclei together with electrons and photons. The latter two are not treated in this paper although the inclusion of them as ideal Fermi and Bose gases respectively are quite simple and now a routine.

The free energy are constructed sums of the free nucleons, which is not contained in nuclei, the light nuclei, which are defined as deuterons $d$, tritons $t$, helions $h$ (=helium 3), and alpha particles $\alpha$, and other heavy nuclei with proton and neutron number $(Z \leq 1000$ and $N \leq 1000)$.

$$
\begin{array}{r}
f=f_{p, n}+\sum_{j=d, t, h, \alpha} n_{j} F_{j}+\sum_{i} n_{i} F_{i}, \\
F_{j / i}=E_{j / i}+M_{j / i}
\end{array}
$$

where $f_{p, n}$ is free energy density of free nucleons, $n_{j / i}$ and $F_{j / i}$ are number density and free energy of individual nucleus, each index $j$ specifies a light nucleus $(d, t, h$ or $\alpha)$ and index $i$ means an heavy nucleus ( $Z \leq 1000$ and $N \leq 1000$ expect the light nuclei) respectively. $E_{i / j}^{t}$ and $M_{i / j}$ are translational energies and mass part of heavy and light nuclei.

The free energy of free nucleons $f_{p, n}$ is calculated by a RMF theory. The model of heavy nuclei $M_{i}=E_{i}^{S h}+E_{i}^{B}+E_{i}^{C}+E_{i}^{S u}$ are based on the liquid drop mass formula composed of $E_{i}^{t}$, shell $E_{i}^{S h}$, bulk $E_{i}^{B}$, Coulomb $E_{i}^{C}$ and surface energies $E_{i}^{S u}$. In constructing the mass formula of heavy nuclei, some points are appropriately taken into account. We assumed the nuclear mass at low densities and temperatures is equal to that of isolated nuclei in vacuum and the shell energy of nuclei are included in the mass formula to reproduce the nuclear abundances of ordinary NSE EOS at low densities. We approximately take into account the effect that the nuclear bulk, shell, Coulomb and surface energies are affect by the free nucleons and electrons at high densities and temperatures. Furthermore the pasta phases near the saturation densities are also taken into account to reproduce the continuous transition to uniform matter. Only the bubble phase are explicitly calculated and other pasta phases are numerically interpolated between normal droplet and bubble phases.

However we use only experimental mass data and the nuclei with shell effects are limited in our previous EOS. Therefore we adopt not only in addition to the experimental mass data [13] but also the theoretical mass data [14] in this version of EOS. Furthermore we did not take into account the temperature dependence of the bulk energies of nuclei with mass data in previous one. We will redefine the bulk and shell energies of the nuclei with mass data.

$$
\begin{aligned}
E_{i}^{B} & =A_{i}\left\{m_{B}+F^{R M F}\left(n_{s i}, T, Z / A\right)\right\} \\
E_{i}^{S h} & = \begin{cases}M_{i}^{\text {data }}-\left[E_{i}^{B}+E_{i}^{C}+E_{i}^{S u}\right]_{\text {vacuum }} & \left(\rho \leq 10^{12} \mathrm{~g} / \mathrm{cm}^{3}\right), \\
\left(M_{i}^{\text {data }}-\left[E_{i}^{B}+E_{i}^{C}+E_{i}^{\text {Su }}\right]_{\text {vacuum }}\right) \times\left(\rho_{0}-\rho\right) /\left(\rho_{0}-10^{12}\right) & \left(\rho>10^{12} \mathrm{~g} / \mathrm{cm}^{3}\right),\end{cases}
\end{aligned}
$$

where $F^{R M F}\left(\rho_{B}, T, Y_{p}\right)$ is the free energy per baryon predicted by the RMF, $n_{s i}$ is nuclear densities of individual nucleus. and $\rho_{0}$ is the saturation density of the system. The last factor $(\rho-$ $\left.10^{12}\right) /\left(\rho_{0}-10^{12}\right)$ means the decay of shell effects at high densities. The formulation of Coulomb $E_{i}^{C}$, surface energies $E_{i}^{S u}$ and translational energies $E_{i / j}^{t}$ are just same as that of previous paper [12]. 


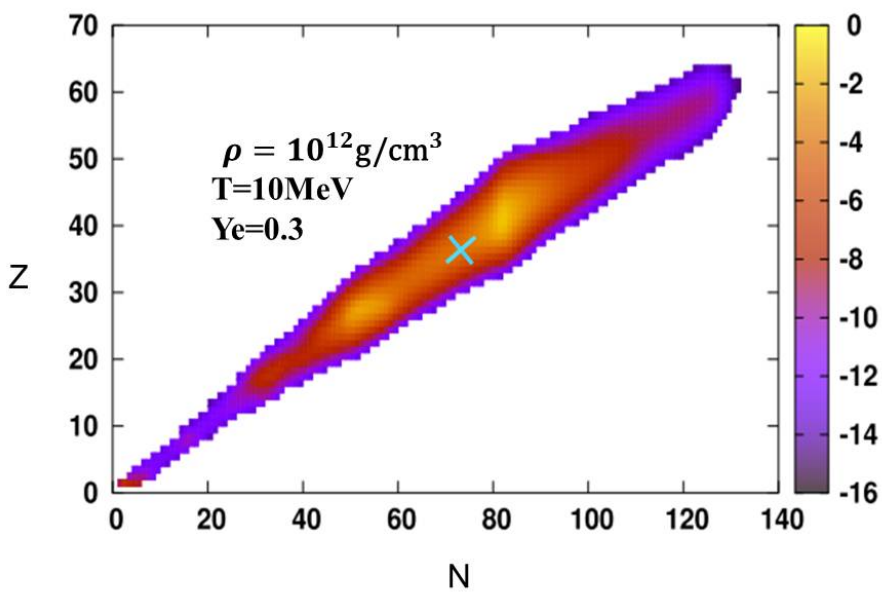

Figure 1: The mass fractions in $\log _{10}$ of nuclei in the $(N, Z)$ plane for $\rho_{B}=10^{12} \mathrm{~g} / \mathrm{cm}^{3}, T=1 \mathrm{MeV}$ and $Y_{p}=$ 0.3 . The cross indicates the representative nucleus for the (H. Shen's EOS 《4) under the same condition.

In our previous EOS, we adopted the liquid drop model even to light nuclei such as deuteron, triton, helion and alpha particles. It is known that the liquid drop mass formula do not reproduce the experimental mass data of the light nuclei, which mass numbers are smaller than about 10 . In our new EOS, the mass of light nuclei $M_{j}$ are approximately calculated by using the shift of binding energies of light nuclei surrounded by dense and hot nucleons provided by Typel et al. [15, 16].

$$
M_{j}=M_{j}^{\text {data }}+\Delta E_{j}^{P a}+\Delta E_{j}^{S . E .}+\Delta E_{j}^{C} \quad(j=d, t, h \& \alpha)
$$

where $\Delta E_{i}^{P a}$ is Pauli energy shift by other baryons, $\Delta E_{j}^{S . E}$ is the self energy shifts of the nucleons composing the light nuclei and $\Delta E_{j}^{C}$ is the coulomb energy shift. The detail explanation of the calculation are provided in Typel et al. [15].

Other parts we modified in this particle from the previous EOS are saturation densities of individual nuclei and the calculation of the excited function of nuclei. Both parts have little influence on the results.

\section{Results}

The mass fractions of nuclei are shown in the $(N, Z)$ plane for $\rho_{B}=10^{12} \mathrm{~g} / \mathrm{cm}^{3}, T=1 \mathrm{MeV}$ and $Y_{p}=0.3$ in Fig. 1 It is clear that nuclei are abundant in the vicinities of the neutron magic numbers $(N=50$ and 82$)$. In fact, the abundance peak arises around $(N, Z) \sim(80,40)$ in our EOS in this case. For the same density, temperature and $Y_{p}$, on the other hand, the Shen's EOS, which is an EOS assuming SNA in wide use for the core-collapse simulation, predicts that the representative nucleus has $(N, Z)=(76,37)$. The difference originates mainly from the fact that the Shen's EOS does not include the shell effect in their Thomas-Fermi approximation. This differences may have an important effect for the electron capture in the collapsing core.

To compare light nuclei abundances $(d, t, h$ and $\alpha)$, we shows that of the ordinary NSE EOS and H. Shen,s EOS other than our model in Fog. 2. In the ordinary NSE EOS, we assumed all 

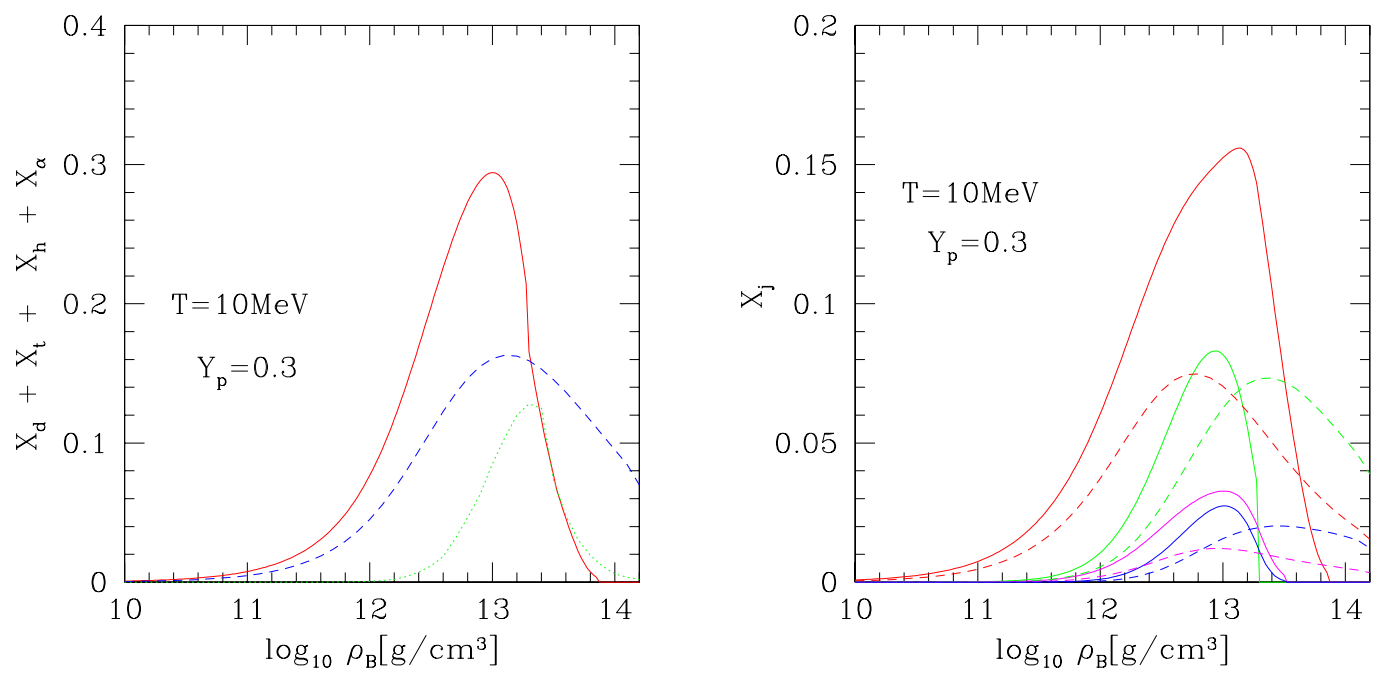

Figure 2: The left panel shows the total mass fractions of light nuclei $(d, t, h, \alpha)$ for our EOS (red solid line) and ordinary NSE (blue dashed line) and the mass fraction of $\alpha$ for H. Shen EOS (green dotted line), in which only alpha particles are included in calculation as representative light nuclei $\left(X_{d}=X_{t}=X_{h}=0\right)$, as a function of density for $T=10 \mathrm{MeV}$ and $Y_{p}=0.3$. The right panel shows the mass fractions of individual light nuclei , $d$ (red), $t$ (green), $h$ (magenta) and $\alpha$ (blue), for our model (solid line) and ordinary NSE (dashed line).

nuclei as the ideal Boltzmann gases which have experimental mass with Coulomb correction $M_{i / j}=$ $M_{i / j}^{\text {data }}+\Delta E_{i / j}^{C}$. H. Shen et al. assume that alpha particle represent the ensemble of light nuclei and light nuclei other than alpha particles are not included in H. Shen's EOS The total mass fraction of the light nuclei, $X_{d}+X_{t}+X_{h}+X_{\alpha}$, of ordinary NSE and our model and that of representative alpha particles of H. Shen EOS $\left(X_{d}=X_{t}=X_{h}=0\right)$ are shown in the left panel Fig. 2 as a function of density for the temperatures $(T=10) \mathrm{MeV}$ and proton fractions $\left(Y_{p}=0.3\right)$. We can see the mass fraction of our EOS shows larger than that of ordinary NSE at the densities $\rho_{B} \lesssim 10^{13} \mathrm{~g} / \mathrm{cm}^{3}$. This is a result of the self-enegy shifts of light nuclei included in our EOS. On the other hand, the Pauli shifts have an important influence on the light nuclei abundances at high densities $\rho_{B} \gtrsim 10^{13} \mathrm{~g} / \mathrm{cm}^{3}$ ,where the abundances are suppressed in our EOS differently from ordinary NSE. H. Shen EOS shows smaller abundances of alpha particle than total abundance of light nuclei in our EOS at almost all densities. This means that the representative alpha particle of H. Shen'S EOS can not representative the ensemble of light nuclei. In a related feature, we can see the deuteron, triton and helion are more abundant than alpha particle in our EOS and ordinary NSE as shown in the right panel Fig. 2. These difference of light nuclei abundances may have an influence on the cooling and heating of shocked matter after bounce in supernovae simulations.

\section{Summary and Discussions}

We have calculated the baryonic equation of state at sub-nuclear densities, which is meant for the use in core-collapse supernova simulations and provides the abundance of various nuclei up to the atomic number of 1000. Basic part of the formulation is same as [12]. The model free 
energy is constructed so that it could reproduce the ordinary NSE results at low densities and make a continuous transition to the EOS for supra-nuclear densities. The experimental and theoretical mass data have been used, whenever available, to obtain the shell energy of nuclei. Assuming the charge neutrality in the W-S cell, we have calculated the Coulomb energy of nuclei. Close to the nuclear saturation density, the existence of the pasta phase has been taken into account in calculating the surface and Coulomb energies. The free energy of the nucleon vapor outside nuclei is calculated by RMF. For light nuclei with $Z \leq 2$ and $N \leq 2$, the quantum approach in exchange for liquid drop approximation are adopted to reproduce the binding energies of that at high densities and temperature.

We find the heavy nuclei abundances are different from the representative nuclei of the EOS assuming SNA and not only alpha particle but also deuteron, triton, helion are abundant in our EOS. These results may affect the dynamics of core-collapse supernova simulations.

\section{Acknowledgments}

This work is partially supported by the Grant-in-Aid for Scientific Research on Innovative Areas (Nos. 20105004, 20105005), the Grant-in-Aid for the Scientific Research (Nos. 19104006, 21540281, 22540296, 24244036) and the HPCI Strategic Program from the Ministry of Education, Culture, Sports, Science and Technology (MEXT) in Japan. S. F. is supported by the Japan Society for the Promotion of Science Research Fellowship for Young Scientists (24 - 79). A part of the numerical calculations were carried out on SR16000 at YITP in Kyoto University.

\section{References}

[1] K. Sumiyoshi, \& G. Röpke, Phys. Rev. C 77 (2008) 055804.

[2] M. Hempel et al., Astrophys. J. 748, (2012) 70.

[3] J. M. Lattimer \& F. D. Swesty, Nucl. Phys., A535, (1991) 331

[4] H. Shen et al. , Nucl. Phys., A637, (1998) 435

[5] H. Shen et al. , Prog. Theor. Phys., 100, (1998) 1013

[6] H. Shen et al. , Astrophys. J. Supplement (2011) 197, 20

[7] A. S. Botvina \& I. N. Mishustin, Phys. Lett. B 584, (2004) 233.

[8] A. S. Botvina \& I. N. Mishustin, Nucl. Phys. A 843, (2010) 98.

[9] J. P. Bondorf et al., K. Phys. Rep. 257, (1995) 133.

[10] M. Hempel \& J. Schaffner-Bielich, Nucl. Phys. A 837,(2010) 210

[11] G. Shen et al., Phys. Rev. C, 83, (2011) 035802

[12] S. Furusawa et al. Astrophys. J. 738, (2011) 178.

[13] G. Audi et al., Nucl. Phys.. A 729, (2003) 337

[14] H. Koura at al. Prog. Theor. Phys. 113,(2005) 305

[15] S. Typel et al. \& Wolter, H. 2010 Phys. Rev. C 81, 015803

[16] G. Röpke Phys. Rev. C 79, (2009) 014002 